\title{
REVISÃO INTEGRATIVA: ADESÃO E NÃO ADESÃO AO REGIME TERAPÊUTICO DE IMUNOSSUPRESSÃO APÓS TRANSPLANTE DE FÍGADO
}

\author{
Integrative review: adherence and non-adherence to the therapeutic immunosuppression \\ regime after liver transplantation
}

Josely Santana Amorim¹, Ângela Aparecida de Lima', André de Souza Silva', Jhon Wesley Fernandes Bragança Miranda²

\section{RESUMO}

O presente estudo objetivou evidenciar adesão medicamentosa ao regime terapêutico imunossupressor em transplantados hepáticos. Para isso, realizou-se uma revisão integrativa da literatura, nos artigos publicados entre 2004 e 2014; os dados foram coletados nos Banco de dados Medline, Scielo, PubMed e BVS, com os descritores: adesão à medicação, medication adherence, cumplimiento a la medicación, transplante de fígado, liver transplantation e transplante hígado. A busca originou 37 artigos; após leitura analítica, resultou em 22 artigos completos. Da análise, emergiram duas categorias: Fatores relacionados à não adesão (NA) e intervenções contribuintes à adesão. Os fatores relacionados à NA foram os riscos psicossociais, abuso de substâncias, baixa adesão ao tratamento e menor conscientização da doença no pré-Transplante (Tx). Já no pós-Tx, os fatores foram gênero masculino e dependente, morar sozinho, idade jovem, maior nível educacional, presença de efeitos colaterais frequentes, tomar medicação diariamente, crenças negativas sobre a medicação e a percepção do Tx e os medicamentos. As intervenções contribuintes à adesão foram: o ajuste da medicação para diminuição da dose e das tomadas diárias, acompanhamento psicológico desde o pré-Tx e assistência farmacêutica no pós-operatório. Concluiu-se que as equipes de saúde devem avaliar aspectos importantes do candidato e implementar estratégias efetivas desde o préTx até o seguimento pós-Tx, a fim de obter melhor adesão ao regime terapêutico imunossupressor no pós-Tx.

Descritores: Transplante de fígado; Adesão à Medicação; Imunossupressão.

Instituições:

${ }^{1}$ Unidade de Transplantes do Hospital das Clínicas da Universidade Federal de Minas Gerais. Belo Horizonte, MG, Brasil

${ }^{2}$ Universidade Federal de Minas Gerais. Escola de Enfermagem da UFMG. Belo Horizonte, MG, Brasil.

\section{Correspondência:}

Josely Santana Amorim

Rua Vitória nº 185, Bairro Niterói, Betim- MG. CEP: 32672-164

Tel: (31) 99119-5848

E-mail: ladyenf@yahoo.com.br
Aceito em: 10/04/2015

\section{INTRODUÇÃO}

$\mathrm{Na}$ atualidade, o transplante de fígado tornou-se uma opção terapêutica eficaz para resolução de moléstias que apresentem o quadro hepatológico irreversível. Segundo a Associação Brasileira de Transplante de Órgãos, durante o ano de 2014 foram realizados 1.755 transplantes hepáticos no Brasil e no primeiro trimestre do ano de 2015 foram realizados 406 transplantes efetuados nos centros credenciados. ${ }^{1,2}$ Esse incremento no número de transplantes ocorrido nos últimos anos é consequência da evolução na área da saúde, que ocorre de forma gradativa, possibilitado pelos avanços nas técnicas cirúrgicas, nos manejos imunológicos e nos cuidados intensivos, além da evolução das drogas imunossupressoras. ${ }^{3}$ 
O propósito do transplante de fígado concentra-se na sobrevivência com qualidade de vida do paciente, sendo este o ponto significativo para que os candidatos submetam-se a tal procedimento. Apesar de ser um tratamento cirúrgico, o transplante distingue-se e destaca-se pela necessidade do uso obrigatório e permanente do medicamento imunossupressor (IS), com o intuito de preservar a funcionalidade e a sobrevida do enxerto e, consequentemente, a vida do paciente transplantado.

Sendo assim, é crucial a aderência do paciente ao protocolo imunossupressor após o transplante (Tx), em virtude das consequências diretas de sua abstenção, que são a rejeição aguda, a disfunção crônica, a perda do enxerto e até mesmo a sua morte. ${ }^{4,5}$ A aderência ao tratamento após o transplante de fígado compreende um dos preceitos fundamentais, que é o cumprimento da terapia medicamentosa imunossupressora. É importante salientar que essa aderência engloba dois conceitos significativos e integrativos, que são: a obediência à dose prescrita e sua forma de administração e, à persistência e duração do tratamento prescrito. ${ }^{6}$

Em consideração a isso, reforça-se que a não aderência (NA) às medicações prescritas é considerada uma das maiores e mais prevalentes causas de perda de enxerto, e esse fato é apresentado, principalmente, em estudos que abordam a aderência dos pacientes transplantados renais. ${ }^{5,7}$ Um desses estudos destacou que a interrupção da rotina diária de tomar a medicação e o ato de esquecimento de tomar alguma dose da medicação foram apontadas como as principais barreiras para a falta de adesão do paciente à terapia imunossupressora. ${ }^{8}$

Outros estudos tiveram o objetivo de conhecer a prevalência, além de identificar fatores que predispõem à adesão e a não adesão terapêutica imunossupressora dos pacientes transplantados renais. ${ }^{9-11}$ Outros estudos apresentaram propostas intervencionistas direcionadas à manutenção do comportamento de aderência ao regime medicamentoso em transplantados renais tardios. $^{12-14}$

No entanto, ainda existem poucos trabalhos voltados para a adesão ao regime imunossupressor em pacientes submetidos ao transplante hepático. O presente estudo tem como objetivo evidenciar a adesão medicamentosa ao regime terapêutico imunossupressor em pacientes submetidos ao transplante de fígado.

\section{MÉTODOS}

Trata-se de uma revisão integrativa da literatura. Os critérios de inclusão definidos para o levantamento dos dados foram artigos que utilizaram os descritores: adesão à medicação, medication adherence e cumplimiento a la medicación associado ao transplante de fígado, liver transplantation e transplante hígado; a busca foi realizada nos Bancos de dados Medline, Scielo, Lilacs, PubMed e BVS, nas publicações que ocorreram no período de 2004 a 2014 e nos idiomas de português, inglês e espanhol. As variáveis de estudo foram relacionadas aos autores, à publicação (fonte, ano de publicação, tipo de estudo) e ao problema de estudo direcionado ao processo de aderência ou não aderência ao regime terapêutico imunossupressor. Foram encontrados 37 artigos indexados nos bancos de dados que relacionava ao tema do estudo, porém a amostra foi constituída de 22 artigos completos, que atendiam aos critérios de inclusão e exclusão.

Foi realizada a leitura analítica sob uma ótica crítica do conteúdo que possibilitou a emersão de duas categorias: fatores relacionados à adesão/nãoadesão e intervenções que contribuem para a adesão medicamentosa ao regime terapêutico imunossupressor em transplantados hepáticos.

\section{RESULTADO E DISCUSSÃO}

Foram utilizadas diversas estratégias metodológicas nos trabalhos utilizados nesse estudo, sendo as principais estratégias o autorrelato, relato de familiares e da equipe de saúde, monitoramento eletrônico, dispensação e contagem de comprimidos e nível sérico das drogas, aplicados de maneira isolada ou combinada.

Os principais tipos de estudo foram o corte transversal e coorte. Nos diversos tipos de estudo, a estratégia mais utilizada foi o autorrelato (A3, A4, A5, A9, A15, A17) onde os resultados demonstraram grandes variações de prevalência de NA em pacientes submetidos ao transplante hepático, entre 22 a $70 \%$. Contrapondo esses achados, alguns autores (A11, A18, A20) apontam índices de NA inferiores a 20\%, com prevalências de 7,1 a $11,5 \%$ entre pacientes do mesmo tipo de Tx.

Observou-se que o estudo da adesão/não adesão à terapia imunossupressora dos pacientes transplantados hepáticos é pouco discutido na literatura, quando comparado a outros tipos de transplante, principalmente o renal. ${ }^{15-36}$

Os artigos foram agrupados em duas categorias (Tabela 1), de acordo com os objetivos propostos, sendo: Fatores relacionados a não adesão ao regime medicamentoso imunossupressor e fatores relacionados a intervenção contribuintes à adesão ao regime medicamentoso imunossupressor. 


\begin{tabular}{|c|c|c|c|c|c|}
\hline \multicolumn{6}{|c|}{ CATEGORIAS } \\
\hline \multicolumn{3}{|c|}{$\begin{array}{c}\text { Fatores relacionados a não adesão } \\
\text { ao regime medicamentoso } \\
\text { imunossupressor }\end{array}$} & \multicolumn{3}{|c|}{$\begin{array}{l}\text { Fatores relacionados a intervenções } \\
\text { contribuintes à adesão ao regime } \\
\text { medicamentoso imunossupressor }\end{array}$} \\
\hline Artigos & Fase pré Tx & Artigos & Fase pós Tx & Artigos & \\
\hline $\begin{array}{l}\text { A1 } \\
\text { A8 }\end{array}$ & $\begin{array}{c}\text { Riscos } \\
\text { psicossociais }\end{array}$ & A1 & $\begin{array}{l}\text { Gênero } \\
\text { masculino e } \\
\text { dependente de } \\
\text { cuidados }\end{array}$ & $\begin{array}{l}\text { A6, } \\
\text { A10, } \\
\text { A11, } \\
\text { A12, } \\
\text { A13 }\end{array}$ & $\begin{array}{l}\text { Ajuste da } \\
\text { medicação } \\
\text { relacionada } \\
\text { às doses e a } \\
\text { diminuição } \\
\text { de tomada } \\
\text { por dia }\end{array}$ \\
\hline $\mathrm{A} 1$ & $\begin{array}{l}\text { Distúrbios de } \\
\text { comportamento }\end{array}$ & A16 & Morar sozinho & $\begin{array}{l}\text { A14, } \\
\text { A18 }\end{array}$ & $\begin{array}{l}\text { Intervenções } \\
\text { farmacêuticas } \\
\text { e psicológicas }\end{array}$ \\
\hline $\begin{array}{l}\text { A8 } \\
\text { A2 }\end{array}$ & $\begin{array}{l}\text { Maior nível } \\
\text { educacional }\end{array}$ & A15 & \multirow[b]{2}{*}{ Idade jovem } & & \\
\hline $\begin{array}{l}\mathrm{A} 8 \\
\mathrm{~A} 1\end{array}$ & $\begin{array}{l}\text { Baixo suporte } \\
\text { social }\end{array}$ & $\begin{array}{l}\mathrm{A} 17 \\
\mathrm{~A} 21\end{array}$ & & & \\
\hline $\begin{array}{l}\text { A1 } \\
\text { A2 } \\
\text { A14 }\end{array}$ & $\begin{array}{l}\text { Baixa adesão } \\
\text { ao tratamento } \\
\text { conservador }\end{array}$ & $\begin{array}{l}\text { A9 } \\
\text { A17 }\end{array}$ & $\begin{array}{c}\text { Ter } \\
\text { experimentado } \\
\text { os efeitos } \\
\text { colaterais da } \\
\text { medicação } \\
\text { de forma } \\
\text { frequente e } \\
\text { intensa. }\end{array}$ & & \\
\hline \multirow[t]{2}{*}{ A8 } & $\begin{array}{c}\text { Menor } \\
\text { conscientização } \\
\text { sobre a doença }\end{array}$ & A11 & $\begin{array}{l}\text { Ter a } \\
\text { obrigatoriedade } \\
\text { de tomar } \\
\text { medicação } \\
\text { diariamente }\end{array}$ & & \\
\hline & & $\begin{array}{l}\text { A16 } \\
\text { A22 }\end{array}$ & $\begin{array}{c}\text { As crenças } \\
\text { negativas sobre } \\
\text { a medicação }\end{array}$ & & \\
\hline $\begin{array}{l}\text { A3 } \\
\text { A4 }\end{array}$ & $\begin{array}{c}\text { Abuso de } \\
\text { substâncias }\end{array}$ & A21 & $\begin{array}{c}\text { A percepção } \\
\text { sobre o } \\
\text { transplante } \\
\text { e os } \\
\text { medicamentos }\end{array}$ & & \\
\hline
\end{tabular}

A categoria Fatores relacionados a não adesão ao regime medicamentoso imunossupressor foi composta por 17 artigos, que discutiam os principais fatores preditores para a NA nas fases de pré-transplante (préTx) e pós-transplante (pós- Tx). Esses estudos apontam indicadores importantes para rastreamento dos possíveis pacientes que podem não aderir ao regime medicamentoso.

No artigo $\mathrm{A} 1$, os autores apontaram que os pacientes transplantados do sexo masculino e dependentes de cuidador, bem como, os que possuíam fatores de risco psicossocial antes do $\mathrm{Tx}$, apresentaram maior predisposição a NA. Além disso, os autores relatam que a presença de distúrbios de comportamento, o baixo suporte social e o maior tempo de Tx podem promover o aumento da NA.

Outros trabalhos (A2 e $\mathrm{A} 14)$ relacionaram que condutas de não adesão ao regime medicamentoso em pacientes na fase pré-Tx, foram preditivas de NA dos imunossupressores no pós-Tx. Também evidenciaram que a adesão destes ao regime terapêutico tende a ser maior até os seis meses pós-Tx, com aumento de NA aos imunossupressores após esse período (entre seis meses até os 36 meses).

Outros fatores no pré-Tx também foram associados à NA no pós-Tx, conforme discutido nos artigos A3, A8 e A21, apontando que o abuso de substâncias, o baixo apoio social, a menor conscientização sobre a moléstia e o maior nível educacional dos candidatos ao Tx são indícios de NA aos imunossupressores no pós-tx.

A presença de efeitos colaterais no uso de imunossupressores foi apontado (A9) como um fator que eleva o número de NA nos pacientes pós-Tx, mostrando que, quanto mais intenso e frequente são os efeitos colaterais mediante ao uso de imunossupressor, maiores são os índices de NA. Já o artigo A19, utilizando metodologia parecida com o anterior, não evidenciou qualquer relação significativa entre a presença de efeitos colaterais e a NA aos imunossupressores. A obrigatoriedade de tomar a medicação diariamente (A11) e a idade mais jovem dos pacientes transplantados (A15, A17 e A21) também foram considerados como principais barreiras que interferem na aderência à terapia medicamentosa imunossupressora no pós-Tx, estando associadas à mudança do estilo de vida e suas restrições.

Os fatores positivos relacionados à adesão aos imunossupressores no pós-Tx, como satisfação e crenças positivas sobre a medicação, foram identificados com maior destaque nos pacientes aderentes (A16, A21), relacionando que a percepção que os pacientes têm sobre seu transplante e medicamentos estão diretamente associados com a adesão à terapia imunossupressora.

Portanto, alguns estudos identificaram na fase pré$T x$, fatores que predispunham à não adesão ao regime medicamentoso imunossupressor no pós $T x$, sendo estes: os riscos psicossociais, os distúrbios de comportamentos, o baixo suporte social, o abuso de substâncias, a baixa adesão ao tratamento clínico e a menor conscientização sobre a doença. ${ }^{15-18,22,28,35}$

Outros estudos apontaram alguns fatores na fase pós-Tx, como transplantados do gênero masculino e dependentes de cuidador, bem como aqueles que moram sozinhos, jovens e com maior nível educacional. A experiência de ter vivenciado algum efeito colateral da medicação de forma frequente e intensa, a conduta obrigatória de tomar as medicações diariamente, as crenças negativas sobre a medicação e a percepção 
do transplante e da medicação contribuem para a não adesão ao regime medicamentoso após a realização do transplante..$^{15,23,25,29-31,35,36}$

Composta por sete artigos, a categoria Fatores relacionados a intervenções contribuintes à adesão ao regime medicamentoso imunossupressor aborda estratégias utilizadas, e que têm contribuído para melhora da adesão medicamentosa em pacientes no pós-Tx. Esses estudos apresentam suas experiências positivas em relação à melhora à adesão à medicação, com o uso da educação em saúde para atingir esse objetivo.

Um fator contribuinte identificado foi o ajuste da medicação relacionada às doses a serem administradas no contexto diário (A6, A7, A10, A11, A12 e A13), utilizando a conversão de tomada de dose do Tacrolimus ${ }^{\circledR}$, intervindo no processo de fragmentação da dose a ser administrada duas vezes ao dia para uma única administração diária, ressaltando ainda que essa conversão é segura. A maioria dos trabalhos discute que essa conversão melhora a adesão aos imunossupressores e não deixa de ser uma opção de intervenção no intuito de favorecer a adesão, evidenciando que ajustar a intensidade do tratamento diário para doses menos frequentes pode ser uma estratégia adequada para minimizar as barreiras para adesão e melhorar a qualidade de vida. Apenas o artigo A7 diverge dessa conclusão.

$O$ atendimento por uma equipe multiprofissional foi relacionado à melhora da adesão medicamentosa. No estudo A18 ficou evidenciado que os pacientes que receberam cuidados farmacêuticos mostraram significativa melhora na adesão aos imunossupressores em relação aos que não receberam tal intervenção. $O$ atendimento por psicólogos foi reforçado como real necessidade, desde o pré-Tx, objetivando favorecer a adesão e até mesmo a não indicação ao transplante para alguns pacientes (A21, A22).

\section{CONCLUSÃO}

O estudo apontou alguns fatores importantes relacionados à adesão/não adesão ao regime terapêutico imunossupressor, englobando tanto o candidato ao transplante como o transplantado de fígado. Também apresentou algumas intervenções de profissionais médicos, farmacêuticos e psicólogos, evidenciando a escassez de condutas no âmbito da enfermagem.

Conclui-se então, que as equipes de saúde devem avaliar aspectos importantes do candidato e implementar estratégias efetivas, desde o pré-transplante até 0 seguimento pós-transplante, a fim de obter melhor adesão ao regime medicamentoso no pós-Tx.

Propõe-se, ainda, que posteriores trabalhos utilizem os principais fatores de não adesão medicamentosa, descritos nesse trabalho, como ferramentas de rastreamento e que se possa conhecer os principais pontos influenciadores na utilização da terapia medicamentosa, sendo uma importante ferramenta de prevenção de saúde, que deve ser amplamente utilizada no acompanhamento ao paciente transplantado.

\section{ABSTRACT}

The current study aimed to demonstrate adherence to the drugs of the therapeutic immunosuppressant regime in liver transplanted individuals. It was performed an integrative review of the literature in articles published between 2004 to 2014. Data were collected on the Medline, Scielo, Pubmed and BVS databases with the following descriptors: medication compliance, medication adherence, cumplimiento a la medicación, transplante de fígado, liver transplantation and transplante hígado. The search originated 37 articles. After an analytical reading, it resulted 22 complete articles. From the analysis, it emerged 2 categories: Factors related to the non-adherence (NA) and interventions contributing to the adherence. Factors related to NA were psychosocial risks, abuse of substances, poor adherence to the treatment, and decreasing awareness of the Disease in the pre transplant period (Tx). As to the post-Tx period, factors analyzed were male gender and dependent, living alone, young, higher educational level, presence of common side effects, intake of the daily medication, negative beliefs on the medication, the perception of the Tx drugs and medicines. The Interventions contributors to the accession have been the adjustment of the medication dose reduction in taken by day, and psychological care from the pre-Tx period and pharmaceutical care in the postoperative period. According to this study, it was concluded that the health care teams should assess major aspects of the candidates and to implement effective strategies from the pre-Tx period to the post-Tx follow-up, in order to attain a higher adherence to the immunosuppressive therapy regime in the post-Tx.

Keywords: Liver transplantation, Medication Adherence, Immunosuppression. 


\section{REFERÊNCIAS}

1. Associação Brasileira de Transplantes de Órgãos. Dados Gerais. Registro Brasileiro de Transplantes. 2013, Ano XIX, Num.4, Jan/Mar.

2. Associação Brasileira de Transplantes de Órgãos. Dados Gerais. Registro Brasileiro de Transplantes. 2014, Ano XIX, Num.1, Jan/Mar.

3. Garcia CD. Manual de doação e transplantes / Clotilde Druck Garcia, Japão Dröse Pereira, Marcelo Kemel Zago, Valter Duro Garcia. - 1. Ed. - Rio de Janeiro: Elsevier, 2013.

4. Nóbrega, RT, Lucena MMS. Para além do transplante hepático: explorando a adesão ao tratamento. Estudos e Pesquisas em Psicologia. 2011,11(3),965-82.

5. Filho MA, Aderência ou concordância: em busca da melhor sobrevida para os transplantes. JBT Jornal Brasileiro de Transplantes. 2007 out/dez,10(4):796-7.

6. Munhoz MID, Andrea TL, Jurado MRP, Chicharro MCC, Villar EM: Adherencia al tratamiento inmusupresor en el paciente adulto con trasplante renal. Enferm Nefrol. 2012 octubre-deciembre;15(4):300-5.

7. McCune KR: Nonadherence to immunosuppressive medication: New insights. The Immunology Report. 2013,10(1):22-5.

8. Schmid-Mohler G, Thut MP, Wüthrich RP, Denhaerynck K, Geest $\mathrm{S}$. Non-adherence to immunoppressive medication in renal transplant recipients within the scope of the integrative model of behavioral prediction: a cross-sectional study. Clin Transplant. 2010,24:213-22.

9. Brahm MMT. Adesão aos imunossupressores em pacientes transplantados renais. Porto Alegre; 2012. Mestrado [Dissertação - Universidade Federal do Rio Grande do Sul, Faculdade de Medicina, Programa de Pós-Graduação em Medicina] - Ciências Médicas.

10. Russel CL, Ashbaugh C, Peace L, Cetingok M, Hamburger $K Q$, Owens S,Coffey D, et al. Time-in-a-bottle (TIAB): a longituginal, correlational study of patterns, potencial predictors, and outcomes of immunosuppressive medication adherence in adult kidney transplant recipients. Clin Transplant. 2013;27:E580-E590.

11. Weng FL, Chandwani S, Kurtyka KM, Zacker C, ChisholmBurns MA. Prevalence and correlates of medication nonadherence among kidney transplant recipients more than 6 months post-transplant: a cross-sectonal study. BMC Nephrology, 2013; 14: 261-270.

12. Chisholm-Burns MA, Spivey CA, Zivin JG, Lee JK, Sredzinski E, Tolley EA. Improving outcomes of renal transplant recipients with behavioral adherence contracts: A randomized controlled trial. American Journal of Transplantation. 2013;13:2364-73.

13. Eisenberger U, Wuthrich RP, Bock A, Ambuhl P, Steiger J, Intondi $A$, et al. Meducation adherence assessment: high accuracy of the new ingestible sensor system in kidney transplants. Transplantation. 2013;96(3):245-50.
14. Chisholm-Burns MA, Spivey CA, Sredzinski E, Butler S. Intervention toolbox to promote immunosuppressant therapy adherence in adult renal transplant recipients. Journal of the American Pharmacists Association. 2012;52(6):816-822.

15. Rodrigue JR; Nelson DR, Hanto DW, Reed AI, Curry MP. Patient-reported immunosuppression nonadherence 6 to 24 months after liver transplant: association with pretransplant psychosocial factors and perceptions of health status change. Prog Transplant. 2013 Dec;23(4):319-28.

16. De Geest S, Burkhalter H, Bogert L, Berben L, Glass TR, Denhaerynck K. Describing the evolution of medication nonadherence from pretransplant until 3 years post-transplant and determining pretransplant medication nonadherence as risk factor for post-transplant nonadherence to immunosuppressives: the Swiss Transplant Cohort Study. Transpl Int. 2014 Jul;27(7):657-66.

17. Lieber SR, Volk ML. Non-adherence and graft failure in adult liver transplant recipients. Dig Dis Sci. 2013 Mar;58(3):824-34.

18. Lamba S, Nagurka R, Desai KK, Chun SJ, Holland B, Koneru B. Self-reported non-adherence to immunesuppressant therapy in liver transplant recipients: demographic, interpersonal, and intrapersonal factors. Clin Transplant. 2012 Mar-Apr;26(2):328-35.

19. De Bleser L, Dobbels F, Berben L, Vanhaecke J, Verleden $G$, Nevens F, et al. The spectrum of nonadherence with medication in heart, liver, and lung transplant patients assessed in various ways. Transpl Int. 2011 Sep;24(9):882-91.

20. Beckebaum S, lacob S, Sweid D, Sotiropoulos GC, Saner F, Kaiser G, et al. Efficacy, safety, and immunosuppressant adherence in stable liver transplant patients converted from a twice-daily tacrolimus-based regimen to once-daily tacrolimus extended-release formulation. Transpl Int. 2011 Jul; 24(7):666-75.

21. Bäckman L, Persson CA. An observational study evaluating tacrolimus dose, exposure, and medication adherence after conversion from twice- to once-daily tacrolimus in liver and kidney transplant recipients. Ann Transplant. 2014;19:138-44.

22. Dobbels F, Vanhaecke J, Dupont L, Nevens F, Verleden G, Pirenne J, et al. Pretransplant predictors of posttransplant adherence and clinical outcome: an evidence base for pretransplant psychosocial screening. Transplantation. 2009 May 27;87(10):1497-504.

23. Wang C; Wang G; Yi H; Tan J; Xu C; Fang X, et al.. Symptom experienced three years after liver transplantation under immunosuppression in adults. PLoS One. 2013 8(11):e80584.

24. Eberlin M; Otto G; Krämer I. Increased medication compliance of liver transplant patients switched from a twice-daily to a once-daily tacrolimus-based immunosuppressive regimen. Transplant Proc. 2013 JulAug; 45(6):2314-20. 
25. Morales JM; Varo E; Lázaro P. Immunosuppressant treatment adherence, barriers to adherence and quality of life in renal and liver transplant recipients in Spain. Clin Transplant. 2012 Mar-Apr;26(2):369-76.

26. Dopazo C; Rodriguez R; Llado L; Calatayud D; Castells L; Ramos $\mathrm{E}$, et al. Successful conversion from twice-daily to once-daily tacrolimus in liver transplantation: observational multicenter study. Clin Transplant. 2012 Jan-Feb;26(1):E32-7.

27. First clinical experience with the new once-daily formulation of tacrolimus. Ther Drug Monit. 2008 Apr;30(2):159-66.

28. Telles-Correia D, Barbosa A, Mega I, Monteiro E. Psychosocial predictors of adherence after liver transplant in a single transplant center in Portugal. Prog Transplant. 2012 Mar;22(1):91-4.

29. Burra P, Germani G, Gnoato F, Lazzaro S, Russo FP, Cillo $\mathrm{U}$, et al. Adherence in liver transplant recipients. Liver Transpl. 2011 Jul;17(7):760-70.

30. Hugon A, Roustit M, Lehmann A, Saint-Raymond C, Borrel E, Hilleret MN, et al. Influence of intention to adhere, beliefs and satisfaction about medicines on adherence in solid organ transplant recipients. Transplantation. 2014 Jul;27;98(2):222-8.

31. Sankaranarayanan J, Collier D, Furasek A, Reardon T, Smith LM, McCartan M, et al. Rurality and other factors associated with adherence to immunosuppressant medications in community-dwelling solid-organ transplant recipients. Res Social Adm Pharm. 2012 May-Jun;8(3):228-39.
32. Klein A, Otto G, Kramer I. Impact of a pharmaceutical care program on liver transplant patients'compliance with immunosuppressive medication: a prospective, randomized, controlled trial using electronic monitoring. Transplantation. 2009 Mar 27;87(6):839-47.

33. Drent G, Moons P, De Geest S, Kleibeuker JH, Haagsma EB. Symptom experience associated with immunosuppressive drugs after liver transplantation in adults: possible relationship with medication non-compliance? Clin Transplant. 2008 Nov-Dec;22(6):700-9.

34. Su GC, Greanya ED, Partovi N, Yoshida EM, Shapiro RJ, Levy RD. Assessing medication adherence in solidorgan transplant recipients. Exp Clin Transplant. 2013 Dec;11(6):475-81.

35. Kung M, Koschwanez HE, Painter L, Honeyman V, Broadbent $\mathrm{E}$. Immunosuppressant nonadherence in heart, liver, and lung transplant patients: associations with medicationbeliefs and illness perceptions. Transplantation. 2012 May 15;93(9):958-63.

36. O'Carroll RE, McGregor LM, Swanson V, Masterton G, Hayes PC. Adherence to medication after liver transplantation in Scotland: a pilot study. Liver Transpl. 2006 Dec;12(12):1862-8. 